Supplement of

\title{
Air quality and health benefits from ultra-low emission control policy indicated by continuous emission monitoring: a case study in the Yangtze River Delta region, China
}

Yan Zhang et al.

Correspondence to: Yu Zhao (yuzhao@nju.edu.cn)

The copyright of individual parts of the supplement might differ from the article licence. 


\section{Table List}

Table S1 Evaluation of WRF simulation performance of meteorological parameters in D2.

Table S2 The ultra-low emission standard limits by sector $\left(\mathrm{mg} / \mathrm{m}^{3}\right)$.

Table S3 The fractions of males and females by region in the YRD for 2015.

Table S4 The baseline mortalities $\left(\mathrm{y}_{0}\right.$, cases $\left./ 10^{5}\right)$ by age group in China.

Table S5 The life expectancy by age group in China.

\section{Figure List}

Figure S1 The spatial distribution of population in the YRD region for 2015 at a horizontal resolution of $9 \mathrm{~km}$ (unit: persons/grid cell). The map data provided by Resource and Environment Data Cloud Platform are freely available for academic use (http://www.resdc.cn/data.aspx?DATAID=201), (C) Institute of Geographic Sciences \& Natural Resources Research, Chinese Academy of Sciences.

Figure S2. The locations and sizes (installed capacity) of coal-fired power plants in the YRD region. 
Table S1 Evaluation of WRF simulation performance of meteorological parameters in D2.

\begin{tabular}{|c|c|c|c|c|c|c|}
\hline Parameters & & Jan & Apr & Jul & Oct & Benchmark \\
\hline \multirow{5}{*}{$\begin{array}{c}\text { Wind speed } \\
\text { (WS10) }\end{array}$} & Obs (m/s) & 2.69 & 2.99 & 2.75 & 2.43 & \\
\hline & $\operatorname{Sim}(\mathrm{m} / \mathrm{s})$ & 2.80 & 3.11 & 2.67 & 2.52 & \\
\hline & Bias (m/s) & 0.12 & 0.11 & -0.08 & 0.09 & \\
\hline & $\mathrm{RMSE}(\mathrm{m} / \mathrm{s})$ & 0.39 & 0.43 & 0.34 & 0.33 & $\leq 2.0$ \\
\hline & IOA & 0.94 & 0.95 & 0.97 & 0.95 & $\geq 0.6$ \\
\hline \multirow{3}{*}{$\begin{array}{l}\text { Wind direction } \\
\text { (WD10) }\end{array}$} & Obs $\left({ }^{\circ}\right)$ & 183.05 & 177.54 & 163.67 & 174.14 & \\
\hline & $\operatorname{Sim}\left(^{\circ}\right)$ & 161.16 & 153.44 & 147.07 & 145.58 & \\
\hline & $\operatorname{Bias}\left({ }^{\circ}\right)$ & -21.90 & -23.32 & -16.60 & -28.56 & $\leq 10$ \\
\hline \multirow{5}{*}{$\begin{array}{c}\text { Temperature } \\
\text { (T2) }\end{array}$} & Obs $\left({ }^{\circ} \mathrm{C}\right)$ & 4.86 & 15.49 & 26.21 & 18.11 & \\
\hline & $\operatorname{Sim}\left({ }^{\circ} \mathrm{C}\right)$ & 4.96 & 15.12 & 25.59 & 18.22 & \\
\hline & $\operatorname{Bias}\left({ }^{\circ} \mathrm{C}\right)$ & 0.10 & -0.35 & -0.62 & 0.12 & $\leq 0.5$ \\
\hline & $\mathrm{RMSE}\left({ }^{\circ} \mathrm{C}\right)$ & 0.70 & 1.06 & 1.13 & 0.63 & \\
\hline & IOA & 0.99 & 0.99 & 0.97 & 0.99 & $\geq 0.7$ \\
\hline \multirow{5}{*}{$\begin{array}{c}\text { Relative } \\
\text { Humidity } \\
\text { (RH2) }\end{array}$} & Obs (\%) & 66.58 & 71.04 & 81.32 & 71.81 & \\
\hline & Sim (\%) & 72.93 & 77.64 & 82.16 & 68.61 & \\
\hline & Bias (\%) & 6.35 & 6.60 & 0.84 & -3.20 & \\
\hline & RMSE (\%) & 13.26 & 10.36 & 3.55 & 6.48 & \\
\hline & IOA & 0.85 & 0.89 & 0.97 & 0.96 & $\geq 0.7$ \\
\hline
\end{tabular}

Note: The benchmarks of statistical indicators for the meteorological parameters were reported in (Emery, C., Tai, E., and Yarwood, G.: Enhanced meteorological modeling and performance evaluation for two Texas episodes, Report to the Texas Natural Resources Conservation Commission, prepared by ENVIRON, International Corp, Novato, CA, 2001). The Bias, IOA and RMSE were calculated using the following equations ( $P, O$ and $\bar{O}$ represent the simulation, observation, and averaged observation value, respectively):

$$
\text { Bias }=\frac{1}{n} \sum_{i=1}^{n}\left(P_{i}-O_{i}\right) ; \quad I O A=1-\frac{\sum_{i=1}^{n}\left(P_{i}-O_{i}\right)^{2}}{\sum_{i=1}^{n}\left(\left|P_{i}-\bar{O}_{i}\right|+\left|O_{i}-\bar{O}_{i}\right|\right)^{2}} ; \quad R M S E=\sqrt{\frac{1}{n} \sum_{i=1}^{n}\left(P_{i}-O_{i}\right)^{2}}
$$


Table S2 The ultra-low emission standard limits by sector $\left(\mathrm{mg} / \mathrm{m}^{3}\right)$.

\begin{tabular}{|c|c|c|c|c|}
\hline Sector & Section & Pollutants & $\begin{array}{c}\text { Ultra-low } \\
\text { emission limits }\end{array}$ & References \\
\hline \multirow{3}{*}{ Power } & \multirow{3}{*}{ Coal-fired } & $\mathrm{SO}_{2}$ & 35 & \multirow{3}{*}{ MEP et al. (2015) } \\
\hline & & $\mathrm{NO}_{\mathrm{X}}$ & 50 & \\
\hline & & PM & 10 & \\
\hline \multirow{3}{*}{ Iron \& steel } & \multirow{3}{*}{ Sintering } & $\mathrm{SO}_{2}$ & 35 & \multirow{3}{*}{ MEE (2019) } \\
\hline & & $\mathrm{NO}_{\mathrm{X}}$ & 50 & \\
\hline & & PM & 10 & \\
\hline \multirow{3}{*}{ Iron \& steel } & \multirow{3}{*}{$\begin{array}{l}\text { Iron } \\
\text { making }\end{array}$} & $\mathrm{SO}_{2}$ & 50 & \multirow{3}{*}{ MEE (2019) } \\
\hline & & $\mathrm{NO}_{\mathrm{X}}$ & 150 & \\
\hline & & $\mathrm{PM}$ & 10 & \\
\hline \multirow{3}{*}{ Cement } & \multirow{3}{*}{ Cement } & $\mathrm{SO}_{2}$ & 30 & \multirow{3}{*}{$\begin{array}{l}\text { HBDEE and HBAMR } \\
(2020)\end{array}$} \\
\hline & & $\mathrm{NO}_{\mathrm{X}}$ & 50 & \\
\hline & & $\mathrm{PM}$ & 10 & \\
\hline \multirow{3}{*}{ Industrial boiler } & \multirow{3}{*}{ Coal-fired } & $\mathrm{SO}_{2}$ & 50 & \multirow{3}{*}{$\begin{array}{l}\text { SDDEE and SDBQTS } \\
\text { (2018) }\end{array}$} \\
\hline & & $\mathrm{NO}_{\mathrm{X}}$ & 100 & \\
\hline & & PM & 10 & \\
\hline
\end{tabular}

Note: The details for the references:

Hebei Department of Ecology and Environment (HBDEE), Hebei Administration for Market Regulation (HBAMR). Ultra-low Emission Standard of Air Pollutants for Cement Industry, DB13/2167-2020. 2020, Hebei, China.

Ministry of Ecology and Environment of the People's Republic of China (MEE), Opinions on Promoting the Implementation of Ultra-low Emissions in the Steel Industry, 2019, Beijing, China.

Ministry of Environmental Protection of the People's Republic of China (MEP), National Development and Reform Commission (NDRC), National Energy Administration (NEA). Work Plan for Fully Implementing Ultra-low Emissions and Energy Conservation in Coal-Fired Power Plants, 2015, Beijing, China.

Shandong Department of Ecology and Environment (SDDEE), Shandong Bureau of Quality and Technical Supervision (SDBQTS). Emission Standard of Air Pollutants for Boilers, DB37/2374-2018. 2018, Shandong, China. 
Table S3 The fractions of males and females by region in the YRD for 2015.

\begin{tabular}{ccc}
\hline Province or city & Male & Female \\
\hline Anhui & 0.51 & 0.49 \\
Shanghai & 0.50 & 0.50 \\
Jiangsu & 0.50 & 0.50 \\
Zhejiang & 0.51 & 0.49 \\
\hline
\end{tabular}


Table S4 The baseline mortalities $\left(\mathrm{y}_{0}, \mathrm{cases} / \mathbf{1 0}^{5}\right)$ by age group in China. Note LRI is a common disease among young children, and its baseline mortalities are 13.7 and 11.4 cases $/ 10^{5}$ for male and female regardless of age, respectively.

\begin{tabular}{ccccccccc}
\hline \multirow{2}{*}{ Age (year) } & \multicolumn{2}{c}{ IHD } & \multicolumn{2}{c}{ STK } & \multicolumn{2}{c}{ COPD } & \multicolumn{2}{c}{ LC } \\
\cline { 2 - 8 } & Male & Female & Male & Female & Male & Female & Male & Female \\
\hline $25-29$ & 5.9 & 2.1 & 4.9 & 1.8 & 0.7 & 0.4 & 1.1 & 0.6 \\
$30-34$ & 9.7 & 3.1 & 7.8 & 2.9 & 1 & 0.7 & 2.5 & 1.3 \\
$35-39$ & 15.3 & 5 & 13.6 & 5.8 & 1.8 & 1.1 & 4.7 & 2.9 \\
$40-44$ & 26.7 & 9.2 & 27.3 & 13.2 & 3.8 & 2.3 & 10.9 & 6.1 \\
$45-49$ & 45.2 & 16.4 & 52.2 & 27 & 7.5 & 4.1 & 25.4 & 11.4 \\
$50-54$ & 77.5 & 29.7 & 100.3 & 52.8 & 17.5 & 8.9 & 55.4 & 21.7 \\
$55-59$ & 125.8 & 50.2 & 176.6 & 89.2 & 39.3 & 18.6 & 102.9 & 35.4 \\
$60-64$ & 215.1 & 99 & 326.5 & 165.4 & 97.8 & 46.2 & 180.3 & 57.1 \\
$65-69$ & 372.6 & 182.9 & 571.1 & 300.5 & 221.6 & 105.2 & 270.5 & 83.1 \\
$70-74$ & 615 & 364 & 996.8 & 553.3 & 494.7 & 238.5 & 381.2 & 126.3 \\
$75-79$ & 1050.4 & 676.3 & 1648.7 & 993.2 & 910.6 & 475.1 & 495.2 & 175.5 \\
$80+$ & 2865.5 & 2551.6 & 3551.5 & 3037.6 & 2459 & 1782.1 & 622 & 268.7 \\
\hline
\end{tabular}


Table S5 The life expectancy by age group in China.

\begin{tabular}{|c|c|c|}
\hline Age (year) & Male & Female \\
\hline$<1$ & 74.8 & 77.7 \\
\hline $1-4$ & 74.5 & 77.4 \\
\hline $5-9$ & 70.6 & 73.5 \\
\hline $10-14$ & 65.7 & 68.6 \\
\hline $15-19$ & 60.8 & 63.7 \\
\hline $20-24$ & 55.9 & 58.8 \\
\hline $25-29$ & 51.1 & 53.9 \\
\hline $30-34$ & 46.2 & 49.0 \\
\hline $35-39$ & 41.5 & 44.2 \\
\hline $40-44$ & 36.7 & 39.4 \\
\hline $45-49$ & 32.0 & 34.6 \\
\hline $50-54$ & 27.3 & 29.9 \\
\hline $55-59$ & 22.9 & 25.3 \\
\hline $60-64$ & 18.6 & 20.9 \\
\hline $65-69$ & 14.8 & 16.8 \\
\hline $70-74$ & 11.4 & 13.2 \\
\hline $75-79$ & 8.7 & 10.0 \\
\hline $80-84$ & 6.7 & 7.5 \\
\hline 85-89 & 4.9 & 5.5 \\
\hline $90-94$ & 3.7 & 4.2 \\
\hline $95-99$ & 2.9 & 3.2 \\
\hline $100+$ & 2.4 & 2.8 \\
\hline
\end{tabular}


Figure S1

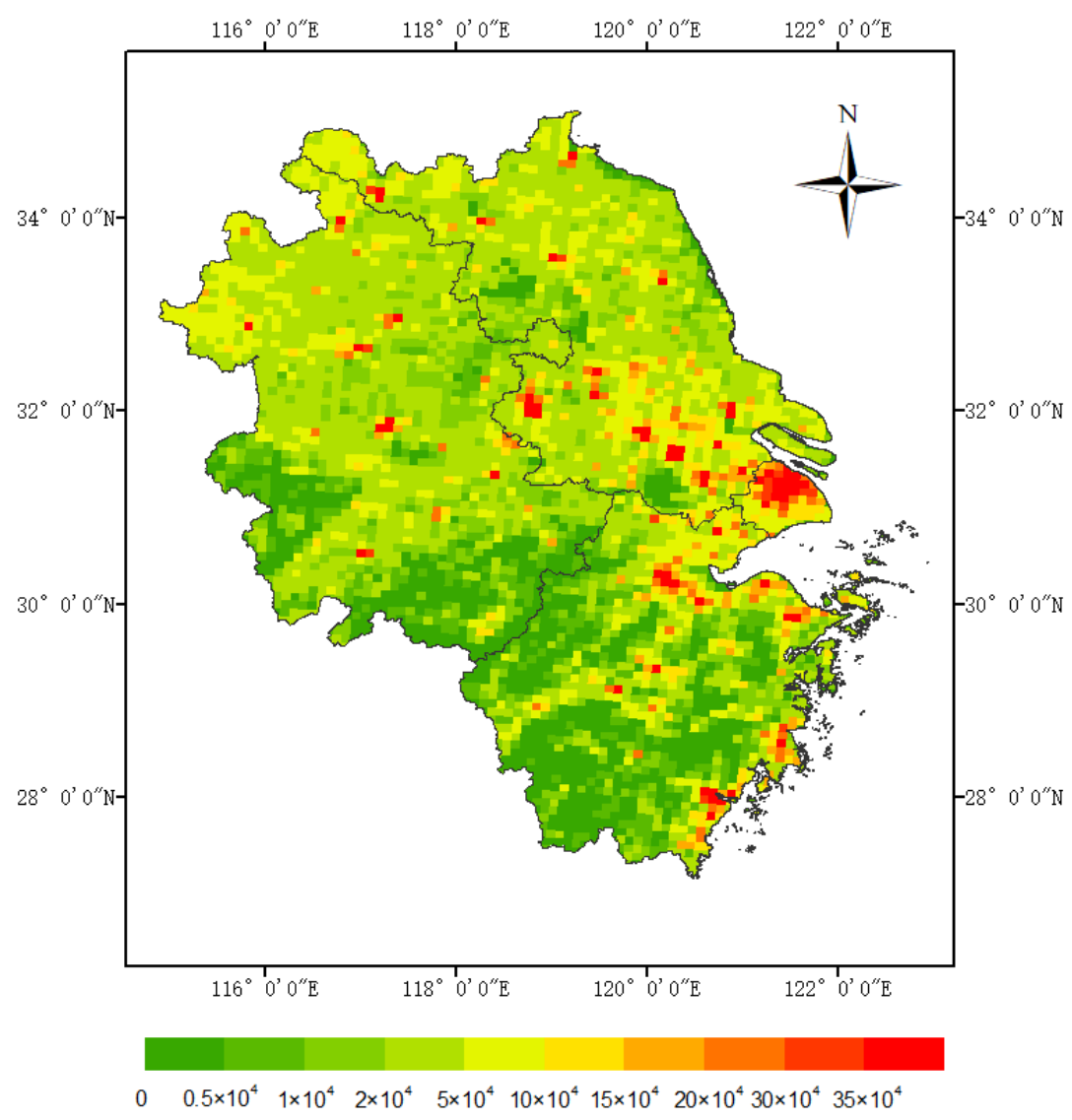


Figure S2



